\title{
Effect of Clonidine versus magnesium sulphate on hemodynamic response to laryngoscopy in day care surgeries: A prospective, randomized, single-center study
}

\author{
Nimisha S. Shiwalkar ${ }^{1,2,3}$ M.D., D.N.B., Akshay P. Kumar ${ }^{1}$ M.D., M.S., M.Ch., Pramod \\ L. Kale ${ }^{2}$ M.D., Vishakha S. Khalde ${ }^{2}$ D.N.B., Vikas Nair ${ }^{2}$ M.D. \\ 1. Department of Anesthesiology, Lokmanya Tilak Municipal Medical College and \\ Hospital, Mumbai, Maharashtra, India \\ 2. Department of Anesthesiology, Joy Hospital Pvt. Ltd, Mumbai, Maharashtra, INdia \\ 3. Department of Anesthesiology, University of Texas Health Science Center, TX, USA
}

Corresponding Author: Dr.Nimisha S. Shiwalkar,

Department of Anesthesiology,

Lokmanya Tilak Municipal Medical College and Hospital,

Sion East, Mumbai, Maharashtra 400022, India

Phone: +91-9967079691,

Email: dr.nimisha4u@gmail.com

\begin{abstract}
Background: Prevention of hemodynamic response to laryngoscopy and intubation is always an integral component of a balanced anesthesia technique and has an escalated significance in the era of day care surgeries. Researchers have always struggled to arrive at a consensus on an ideal drug and its dose to control the laryngoscopy response. As such a drug with more physiological pharmacodynamic profile, magnesium sulfate, can be advantageous in majority of cases. We compare it with another potent drug, clonidine, an alpha2 agonist, which has proven its role in attenuating laryngoscopic response in earlier studies.

Methods: A single-centre prospective randomized double-blinded study was conducted on 90 patients with ASA grade 1 and 2 undergoing elective laparoscopic cholecystectomy using general anaesthesia. Patients were randomly assigned to receive either MgSO4 (elemental Magnesium 50mg/kg), clonidine 2 micrograms $/ \mathrm{kg}$ or normal saline (NS) prior to induction and evaluated for hemodynamic responses to laryngoscopy. Data was compared using Oneway ANOVA, Kruskal-Wallis and Chi-Square tests.

Results: Clonidine group was better than magnesium sulphate group in attenuating the rise in heart rate associated with laryngoscopy while magnesium sulphate and clonidine were equally effective in controlling the rise in blood pressure associated with laryngoscopy.

Conclusion: Both IV Magnesium sulphate $50 \mathrm{mg} / \mathrm{kg}$ and IV Clonidine $2 \mathrm{mcg} / \mathrm{kg}$ given prior to the induction of anesthesia in laparoscopic surgery effectively attenuated the hemodynamic response to laryngoscopy with Clonidine being better than magnesium sulphate in controlling the rise heart rate associated with laryngoscopy.
\end{abstract}

Keywords: Laryngoscopy, Laparoscopy, Magnesium sulphate, Clonidine, alpha2 agonists, anesthesia 


\section{Introduction}

Laryngoscopy and tracheal intubation are of paramount importance during conduct of general anesthesia. Different drugshave beentested over the years to overcome or attenuate this response but an ideal agent still remains elusive.The anatomical and physiological understanding of the laryngoscopic response reveals an underlying reflex sympathetic discharge caused by epipharyngeal and laryngopharyngeal stimulationcausing hypertension, tachycardia and arrhythmias ${ }^{1,2}$.The increase in blood pressure and heart rate thoughtransient are usually variable and unpredictable.Hypertensive patients areparticularly vulnerable to this sudden and significant increase in BP. Transitory hypertension and tachycardia are probably of no consequence in healthy individuals but may prove to be hazardous in those with hypertension, ischemic heart disease or cerebrovascular disease or a pre-existing increased intracranial or intraocular pressure, predisposing them to the development of pulmonary edema,myocardial insufficiencyand cerebrovascular accident ${ }^{32,} 4,{ }^{5}$.Such conditions necessitate blunting of these harmful laryngoscopic responses.

Many pharmacological methods have been devised to reduce the extent of hemodynamic events like high dose of opioid,local anesthetics like lignocaine, alpha and betaadrenergic blockersand vasodilatation drugs like nitroglycerine ${ }^{6-8}$.Topical anesthesia with lignocaine applied to the larynx and trachea in a variety of ways remains a popular method used alone or in combination with other techniques ${ }^{6}$. This study intended to compare the effect of magnesium, which is an essential nutrient for normal body function, on the hemodynamic response to laryngoscopy. We compared itwith an alpha2 agonist clonidine which has been previously used for this purpose.

Magnesium sulphate blocks the release of catecholamines from the adrenergic nerve terminals and adrenal glands in vitro. Moreover, it produces vasodilation by actingdirectly on blood vessels, and also on coronaries ${ }^{9}$. High dose magnesium attenuates vasopressin stimulated vasoconstriction and normalizes sensitivity to vasopressin ${ }^{10}$.Magnesium sulphate also blocks the NMDA receptors in CNS \& thereby produce pain relief ${ }^{11}$. We therefore hypothesized, that Magnesium sulphate might attenuate the hemodynamic stress responses to laryngoscopy in one of the commonest day-care surgery procedures performed worldwide laparoscopic cholecystectomy.We compared its response with an alpha-adrenergic agonist, clonidine, which has a beneficial effect of blunting hemodynamic responses due to laryngoscopy $\&$ is also shown to decrease post-operative pain.

\section{Methodology \\ Study design:}

After obtaining approval from the institutional review board (IRB), we investigated 90 patients between age 18 to 60-year-old, ASA Class I and II undergoing elective laparoscopic cholecystectomy under general anesthesia. Patients with uncontrolled hypertension, diabetes mellitus, severe cardiac, hepatic, respiratory or renal disease, history of allergy to any of the study drugs, pregnancy or breast-feeding females, history of opioid or alcohol intake in last 24 hours, patients receiving Alpha 2 agonists and Beta Blocker and other medical, psychiatric, or behavioral limitations that could interfere with study methodology were excluded. Surgeries which could not be completed laparoscopically and required open cholecystectomy were excluded from the study too.

Patients were randomly divided into three groups: Magnesium sulphate (elemental Magnesium $50 \mathrm{mg} / \mathrm{kg})$, clonidine $(2 \mathrm{ug} / \mathrm{kg})$ or Normal saline by computer-generated randomization table. The details of randomization remained with the operating room pharmacist who issued the drug in identical looking bags of $100 \mathrm{ml}$ Normal Saline (NS). After thorough preoperative checkup, procedure was explained to each patient \& written consent taken. The evaluators remained blinded to the drug and received the drug from the operating 
room pharmacy. All patients were shifted to recovery and under continuous monitoring of heart rate, rhythm and blood pressure, received the drug, with the detailed randomization code staying with the operating room pharmacy.

After premedication with intravenous Midazolam $0.03 \mathrm{mg} / \mathrm{kg}$, patients were induced with Inj. Fentanyl $1 \mathrm{ug} / \mathrm{kg}$ and Inj. Propofol $2 \mathrm{mg} / \mathrm{kg}$, in incremental doses until loss of consciousness. Bispectral index (BIS) was maintained between 40-60 to prevent any hemodynamic changes arising due to awareness. Inj. Rocuronium Bromide $0.7 \mathrm{mg} / \mathrm{kg}$ was administered and laryngoscopy was performed after 90 seconds to facilitate endotracheal intubation. Thereafter, anesthesia was maintained with Isoflurane $1-1.5 \%$ in combination with $50 \%$ air in oxygen, on controlled mechanical ventilation in a closed circuit. Ventilation of the lungs was adjusted to maintain an end-tidal $\mathrm{CO} 2$ of $30+/-5 \mathrm{mmHg}$. Muscle relaxation was maintained using intermittent Rocuronium dosages of $0.15 \mathrm{mg} / \mathrm{kg}$ as per the recordings by peripheral nerve stimulator. Hemodynamic parameters monitored were Heart rate(HR), Electrocardiogram(ECG), Systolic Blood Pressure(SBP), Diastolic Blood Pressure(DBP), Mean Arterial Pressure(MAP), End tidal carbon-dioxide(etCO2) and Peripheral Oxygen saturation(SPO2). These were measured at baseline; prior to injection of drugs; after the injection of drugs; after induction; immediately after laryngoscopy; at 5 minutes and 10 minutes after laryngoscopy.

Any untoward side effects like bradycardia ( $\mathrm{HR}<60$ or if reduced by $20 \%$ than baseline, whichever was less) lasting $>1$ minute was noted and treated with injection Atropine $0.6 \mathrm{mg}$ IV bolus, hypotension (MAP reduced by $20 \%$ of the baseline MAP) for duration of $>1$ min was treated with titration of Isoflurane inhalation and if persisted, treated with injection Mephentermine sulphate $3 \mathrm{mg}$ IV bolus, exaggerated hypertension SBP $>180 \mathrm{mmHg}$, DBP $>110 \mathrm{mmHg}$ was treated with Injection Metoprolol 1mg bolus.

\section{Statistical Analysis:}

A power analysis was performed before the study began. The analysis suggested that 30 patients in each group would be adequate for statistical significance in this study. Data collected was entered in Excel sheet and data analysis was done usingSPSS Software ver 25.0 and Sigmaplot Ver 13. Quantitative data was reported as Mean, SD, Median and IQR, while comparison among study group was done with the help of One-way ANOVA or Kruskal Wallis test as per the results of Normality test. Qualitative data was presented as Frequency and Percentage table, whereas comparison among study group was done with the help of ChiSquare test. A probability value less than 0.05 is taken as significant.

\section{Results}

\begin{tabular}{|l|l|l|l|}
\hline Table 1 & MgSO4 & Clonidine & Control \\
\hline Age (years) & 43 & 46.47 & 43 \\
\hline Weight (kilograms) & 64 & 63.3 & 67.73 \\
\hline Male: Female & 0.66 & 0.5 & 0.5 \\
\hline Heart rate (per minute) & 85.9 & 83.8 & 83.1 \\
\hline Systolic BP (mm of Hg) & 126.1 & 128.97 & 126.87 \\
\hline Diastolic BP (mm of Hg) & 76.03 & 76.93 & 74.47 \\
\hline Mean BP (mm of Hg) & 93.37 & 92.43 & 93.9 \\
\hline Intra-operative EtCO2 (mmHg) & 30.35 & 30.77 & 30.51 \\
\hline
\end{tabular}


The three study groups A (MgSO4), B (Clonidine) and C (Normal Saline-Control) were comparable in terms of age, sex and weight; baseline hemodynamic parameters including heart rate, systolic BP, diastolic BP and Mean BP (Table 1).

Though there was no significant difference in heart rate between the three groups after induction, Group A and Group B were individually better than Group C in attenuating the rise in heart rate at the time of laryngoscopy.Among the two, Group B was better than Group A in attenuating the rise in heart rate immediately following laryngoscopy and Group B also had lower heart rates compared to Group $\mathrm{C}$ at $5 \mathrm{~min}$ after laryngoscopy.No such difference is seen between Group A and Group C at 5min after laryngoscopy. At 10 minutes following laryngoscopy, no significant differences in heart rate were noticed between either of the groups(Figure 1).
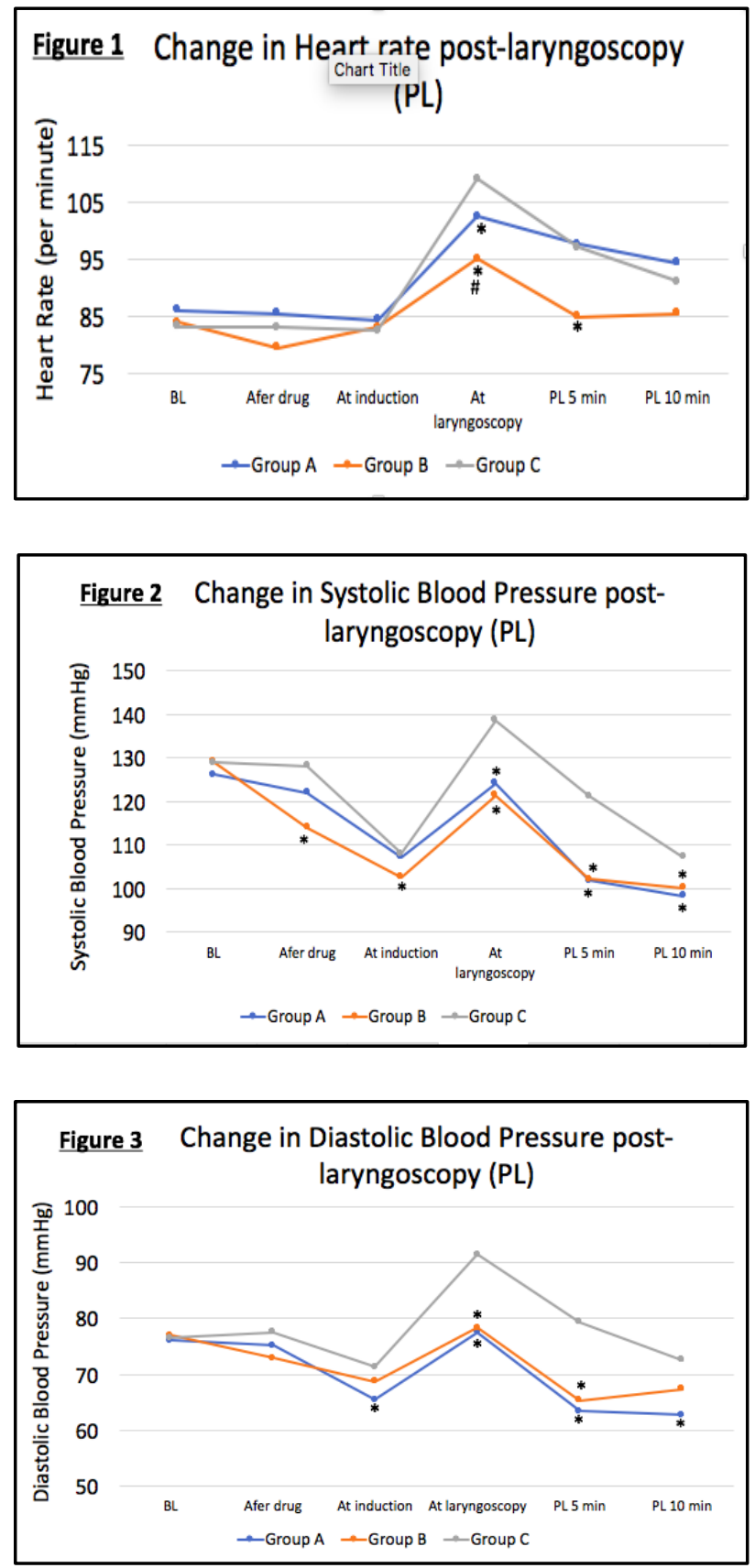


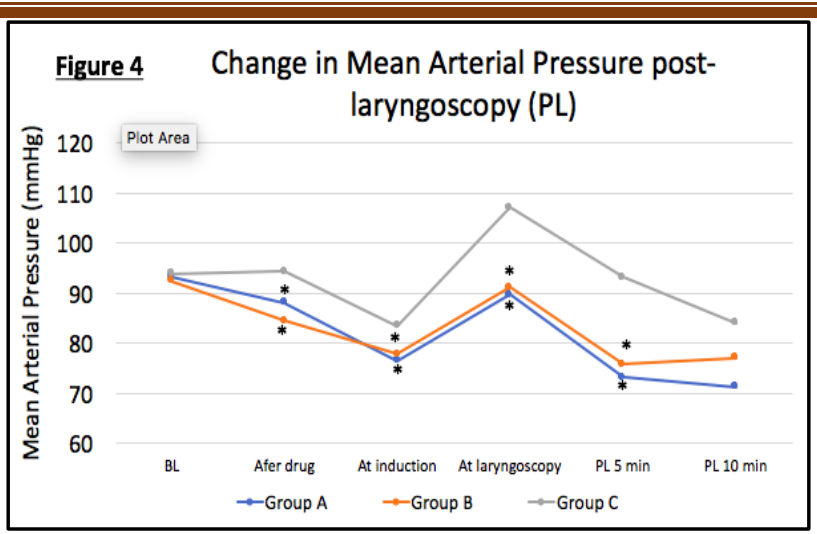

When compared to the systolic BP in Group C, Group B showed a decrease in systolic $\mathrm{BP}$ after the drug was infused and after induction while the systolic BP in Group A remained more or less similar to Group C.Group A and Group B both were better in attenuating the rise in systolic BP immediately, at $5 \mathrm{~min}$ and $10 \mathrm{~min}$ after laryngoscopy with no significant difference between Group A and Group B(Figure 2). Group A had a lower diastolic BP as compared to Group $\mathrm{C}$ following induction. Group A was better than Group $\mathrm{C}$ in attenuating the rise in diastolic BP from the time of laryngoscopy until about 10min while Group B was better than Group C until 5min after laryngoscopy.No significant difference was seen between Group A and Group B at any of the time points(Figure 3). Both Group A and B showed a lower $\mathrm{BP}$ as compared to group $\mathrm{C}$ right after infusion of the respective drugs and following induction. Again, both Group A and B both were better than Group C in attenuating the rise in MAP associated with laryngoscopy up to $5 \mathrm{~min}$. The mean arterial pressures were similar in all groups by $10 \mathrm{~min}$ after laryngoscopy. No significant difference was seen between Group A and Group B(Figure 4).

During the 10-minute period following laryngoscopy, none of the patients required atropine, 1 patient in clonidine group required IV mephenterine and 2 patients in control group required IV metoprolol.

\section{Discussion}

The laparoscopic approach to abdominal surgeries was a milestone achievement in modern era of surgical practice. Today laparoscopic surgeries have been an integral component of ambulatory and day care procedures and require a stable anesthesia technique leading in to a smooth post-operative recovery. One of the major components to ensure this isby avoiding physiological alterations in hemodynamics during laryngoscopy and accompanying cardiovascular perturbationsduring intubation.Manipulation of the respiratory tract during laryngoscopy and tracheal intubation are associated withhemodynamic and cardiovascular responses arising from increased circulating catecholamines and increased sympathetic drive ${ }^{12,13}$. Over the years many studies were carried out using premedication with lidocaine,alpha- agonists, opioidsetc. to attenuate this response.Clonidine is a centrally acting alpha adrenoreceptor agonist with high affinity for alpha2 receptors present mainly post-junctionally in the medulla. The resultant effect of it is a decrease in sympathetic outflow from the central nervous system causing decreased peripheral resistance, heart rate and blood pressure ${ }^{14}$.Magnesium, via calcium antagonism and decreased activity of calmodulin dependent light chain kinase, exerts its peripheral vasodilatory effects ${ }^{15}$. Additional effects of magnesium administration include decreased contractility of myocardium and possibly blockage of sympathetic ganglia to produce a decrease in blood pressure $^{16}$. 
Prior research groups have demonstrated advantage of using clonidine against normal saline $^{17}$ in controlling the rise in heart rate following intubation. An increased heart rate is especially detrimental in patients with a poor cardiac reserve as tachycardia may impair ventricular filling further to decrease cardiac output while increasing the oxygen demand on the other hand. When we compared $2 \mathrm{ug} / \mathrm{kg}$ dose ofclonidine against $50 \mathrm{mg} / \mathrm{kg} \mathrm{MgSO} 4$, we found that clonidine could maintain a relatively stable heart rate uptill 5 minutes following intubation. Magnesium sulphate, though was effective in controlling the heart rate immediately following intubation, it was still inferior to clonidine when followed over next 5 minutes. Magnesium is known to block the acetylcholine release and decrease the vagal tone in prior literature ${ }^{18,19}$. This can be a reason for magnesium to not be able to control the heart rate in our experiment. However, the heart rate in magnesium group never increased more than control group.Honarmand et al. and Nooraei et al. showed that magnesium sulphate $(50 \mathrm{mg} / \mathrm{kg})$ did not confer any advantage over control or lidocaine groups respectively on HR control 1 minute subsequent to laryngoscopy and beyond ${ }^{18,20}$. However, in both these studies the drug was given after induction while we gave the drug prior to induction thereby giving more time for the drug onset of action. On the other hand, Mahajan and colleagues observed that the laryngoscopy induced hemodynamic variation was better controlled while maintaining a BIS of 40-50 which synchronizes with our observation ${ }^{21}$.

We observed that both MgSO $450 \mathrm{mg} / \mathrm{kg}$ and Clonidine $2 \mathrm{ug} / \mathrm{kg}$ were effective in controlling the rise in systolic, diastolic as well as mean arterial pressures following laryngoscopy but clonidine accounted for even lower values. The stress response of laryngoscopy increases the myocardial demand and cardiac afterload through sympathetic and vasoconstrictor responses. The attenuation of rise in blood pressure is essential in controlling these detrimental responses that follow laryngoscopy and intubation. Our data depicting the advantage of using clonidine over normal saline for preventing the intubation response was similar to other studies ${ }^{17,22}$. Similar to our findings, Mahajan et al. ${ }^{21}$ did notice an attenuation in blood pressure recordings following laryngoscopy in patients administered MgSO4. As per our knowledge, no studies comparing the two drugs have been done yet.

Our study compares two commonly used drugs which are heavily investigated in literature to determine their use in attenuating the pressor response to laryngoscopy. We arrive at a conclusion that both these drugs are beneficial in attenuating the rise in blood pressure following laryngoscopy with a slightly better blood pressure control in the clonidine group. However, clonidine showed a better control of heart rate as compared to MgSO4. Further studies would be required to determine whether this clonidine induceddecrease in blood pressure or heart rate is significant enough to require treatment.

\section{Conclusion:}

With better control of heart rate, clonidine $2 \mathrm{ug} / \mathrm{kg}$ may be a better alternative to $\mathrm{MgSO} 4$ in controlling the hemodynamic instability to laryngoscopy, though both the drugs show equal efficacy in controlling the rise in blood pressure following laryngoscopy.

\section{Bibliography:}

1. Kovac AL. Controlling the hemodynamic response to laryngoscopy and endotracheal intubation. Journal of clinical anesthesia. 1996; 8: 63-79.

2. Prys-Roberts C, Greene LT, Meloche R and Foex P. Studies of anaesthesia in relation to hypertension. II. Haemodynamic consequences of induction and endotracheal intubation. British journal of anaesthesia. 1971; 43: 531-47.

3. Fox EJ, Sklar GS, Hill CH, Villanueva R and King BD. Complications related to the pressor response to endotracheal intubation. Anesthesiology. 1977; 47: 524-5. 
4. Maassen RL, Pieters BM, Maathuis B, et al. Endotracheal intubation using videolaryngoscopy causes less cardiovascular response compared to classic direct laryngoscopy, in cardiac patients according a standard hospital protocol. Acta anaesthesiologica Belgica. 2012; 63: 181-6.

5. Perkins ZB, Wittenberg MD, Nevin D, Lockey DJ and O'Brien B. The relationship between head injury severity and hemodynamic response to tracheal intubation. The journal of trauma and acute care surgery. 2013; 74: 1074-80.

6. Thompson KR and Rioja E. Effects of intravenous and topical laryngeal lidocaine on heart rate, mean arterial pressure and cough response to endotracheal intubation in dogs. Veterinary anaesthesia and analgesia. 2016; 43: 371-8.

7. El-Shmaa NS and El-Baradey GF. The efficacy of labetalol vs dexmedetomidine for attenuation of hemodynamic stress response to laryngoscopy and endotracheal intubation. Journal of clinical anesthesia. 2016; 31: 267-73.

8. Channaiah VB, Kurek NS, Moses R and Chandra SB. Attenuation of Hemodynamic Response to Laryngoscopy and Endotracheal Intubation with Pre Induction IV Fentanyl Versus Combination of IV Fentanyl and Sub Lingual Nitroglycerin Spray. Medical archives (Sarajevo, Bosnia and Herzegovina). 2014; 68: 339-44.

9. Herroeder MDS, Schönherr MDDESAME, De Hert MDPDSG and Hollmann MDPDDEAAMW. Magnesium-Essentials for Anesthesiologists. Anesthesiology. 2011; 114: 971-93.

10. Lord MS and Augoustides JG. Perioperative management of pheochromocytoma: focus on magnesium, clevidipine, and vasopressin. Journal of cardiothoracic and vascular anesthesia. 2012; 26: 526-31.

11. Mentes O, Harlak A, Yigit T, et al. Effect of intraoperative magnesium sulphate infusion on pain relief after laparoscopic cholecystectomy. Acta anaesthesiologica Scandinavica. 2008; 52: 1353-9.

12. King BD, Harris LC, Jr., Greifenstein FE, Elder JD, Jr. and Dripps RD. Reflex circulatory responses to direct laryngoscopy and tracheal intubation performed during general anesthesia. Anesthesiology. 1951; 12: 556-66.

13. Stoelting RK. Circulatory response to laryngoscopy and tracheal intubation with or without prior oropharyngeal viscous lidocaine. Anesthesia and analgesia. 1977; 56: 61821.

14. Ronald Miller LE, Lee Fleisher, Jeanine Wiener-Kronish, Neal Cohen, William Young. Miller's Anesthesia. 8 ed. Philadelphia: Elsevier/Saunders, 2014.

15. Euser AG and Cipolla MJ. Magnesium sulfate for the treatment of eclampsia: a brief review. Stroke. 2009; 40: 1169-75.

16. Nakaigawa Y, Akazawa S, Shimizu R, et al. Effects of magnesium sulphate on the cardiovascular system, coronary circulation and myocardial metabolism in anaesthetized dogs. British journal of anaesthesia. 1997; 79: 363-8.

17. Hussain SY, Karmarkar A and Jain D. Evaluation and Comparison of Clonidine and Dexmedetomidine for Attenuation of Hemodynamic Response to Laryngoscopy and Intubation: A Randomized Controlled Study. Anesthesia, essays and researches. 2018; 12: 792-6.

18. Nooraei N, Dehkordi ME, Radpay B, Teimoorian H and Mohajerani SA. Effects of intravenous magnesium sulfate and lidocaine on hemodynamic variables following direct laryngoscopy and intubation in elective surgery patients. Tanaffos. 2013; 12: 57-63.

19. Fawcett WJ, Haxby EJ and Male DA. Magnesium: physiology and pharmacology. British journal of anaesthesia. 1999; 83: 302-20.

20. Honarmand A, Safavi M, Badiei S and Daftari-Fard N. Different doses of intravenous Magnesium sulfate on cardiovascular changes following the laryngoscopy and tracheal 
intubation: A double-blind randomized controlled trial. Journal of research in pharmacy practice. 2015; 4: 79-84.

21. Mahajan L, Kaur M, Gupta R, Aujla KS, Singh A and Kaur A. Attenuation of the pressor responses to laryngoscopy and endotracheal intubation with intravenous dexmedetomidine versus magnesium sulphate under bispectral index-controlled anaesthesia: A placebo-controlled prospective randomised trial. Indian journal of anaesthesia. 2018; 62: 337-43.

22. Sarkar A, Tripathi RK, Choubey S, Singh RB and Awasthi S. Comparison of effects of intravenous clonidine and dexmedetomidine for blunting pressor response during laryngoscopy and tracheal intubation: A randomized control study. Anesthesia, essays and researches. 2014; 8: 361-6. 